\title{
Paroxetine suppresses reactive microglia-mediated but not lipopolysaccharide-induced inflammatory responses in primary astrocytes
}

\section{Xiong Zhang}

Wenzhou Medical University Second Affiliated Hospital

Lan-Bing Zhu

Wenzhou Medical University Second Affiliated Hospital

Jia-Hui He

Wenzhou Medical University Second Affiliated Hospital

Hong-Qiu Zhang

Wenzhou Medical University

Shu-Ya Ji

Wenzhou Medical University

Chao-Nan Zhang

Wenzhou Medical University

$\mathrm{Na}-\mathrm{Na} \mathrm{Hou}$

Wenzhou Medical University

Chen-Ping Huang

Wenzhou Medical University

Jian-Hong Zhu ( $\nabla$ jhzhu@wmu.edu.cn )

Wenzhou Medical University https://orcid.org/0000-0001-9412-8264

\section{Research}

Keywords: paroxetine, astrocytes, microglia, neuroinflammation, Parkinson's disease

Posted Date: November 18th, 2019

DOl: https://doi.org/10.21203/rs.2.17327/v1

License: (c) (i) This work is licensed under a Creative Commons Attribution 4.0 International License. Read Full License 


\section{Abstract}

Background Astrocytes are the most abundant glial cells in a brain that mediate inflammatory responses and provide trophic support for neurons. We have previously disclosed that paroxetine, a common selective serotonin reuptake inhibitor, ameliorates LPS-induced microglia activation. However, it remains elusive of the role of paroxetine in astrocytic responses.

Methods Isolated primary astrocytes were pretreated with paroxetine and stimulated with different stimuli, lipopolysaccharide (LPS) or microglia conditioned medium pre-activated with LPS (M/Lps). Inflammatory and neurotrophic responses, underlying mechanisms and the impact on neuronal survival were assessed.

Results Paroxetine had no impact on LPS-stimulated iNOS, TNF- $a$ and IL-1 $\beta$ expression, but inhibited M/Lps-induced TNF- $a$ and IL-1 $\beta$ expression in primary astrocytes. Paroxetine suppressed M/Lps- but not LPS-induced activation of NF-KB and had no impact on activation of MAPKs and STAT3. Incubation with the resulted astrocyte conditioned media caused no change in viability of SH-SY5Y cells. BDNF and MANF mRNA expressions were upregulated by M/Lps and paroxetine, respectively. However, M/Lps- or LPS-induced extracellular releases of NO, TNF-a and/or BDNF in astrocytes were in minor amount compared to those by microglia.

Conclusions Paroxetine ameliorates the reactive microglia-mediated inflammatory responses in astrocytes partially via inhibition of NF-KB pathway, but has no impact on LPS-stimulated astrocyte activation. While the secondary astrocytic responses are not robust compared to the innate immune responses of microglia, our results support a therapeutic potential of paroxetine against neuroinflammation-associated neurological disorders such as Parkinson's disease.

\section{Background}

Parkinson's disease (PD) is a common neurodegenerative disease characterized by the selective death of dopaminergic neurons in the substantia nigra. Although its exact etiology remains elusive, accumulating evidence has suggested that neuroinflammation plays a key role in the pathogenesis of PD [1-3]. In the central nervous system, innate immune responses are collectively mediated by microglia and astrocytes while the former dominates the way to neuroinflammation [4,5]. Indeed, reactive microglia and astrocytes are both found in the nigrostriatal bundle of PD patients or PD animal models [6-9]. Different from microglia, reactive astrocytes depending on the context may release pro-inflammatory factors such as tumor necrosis factor a (TNF- $\alpha$ ) and interleukin $-1 \beta(\mathrm{IL}-1 \beta)$, or provide trophic support for neurons by releasing neurotrophic factors such as brain derived neurotrophic factor (BDNF) and mesencephalic astrocyte-derived neurotrophic factor (MANF) $[4,5,10]$. The interaction between microglia and astrocytes leads to collective outcomes to neurons. For instance, astrocytes were reported to enhance the inflammatory responses of activated microglia, resulting in more dopaminergic neuron death [11]. 
Studies have suggested negative immunoregulatory effects of antidepressant drugs [12-15]. Amongst, paroxetine is a common selective serotonin reuptake inhibitor, and is shown to protect against dopaminergic neuronal loss in a MPTP-induced mouse model of PD, potentially through its inflammatory alleviation in the substantia nigra [16]. By applying lipopolysaccharide (LPS), an endotoxin often used for modeling PD in the context of neuroinflammation $[17,18]$, we have previously disclosed that paroxetine ameliorates LPS-induced microglia activation through regulation of MAPK signaling [19]. However, it remains elusive of the role of paroxetine in astrocytic responses. Thus, in this study we aimed to understand the impact of paroxetine on astrocyte activation induced by LPS and reactive microglia using a conditioned medium culture system.

\section{Methods}

\section{Cell culture and primary astrocyte isolation}

Dulbecco's modified eagle medium (DMEM; C11995500BT) and DMEM/F-12 (C11330500BT) were purchased from Gibco (Grand Island, NY, USA). Fetal bovine serum (FBS; VS500T, Australia origin) was from Ausbian (Shanghai, China). BV2 microglia cells (provided by Dr. Zhu CQ, Fudan University) and SHSY5Y cells (Cell Bank of Chinese Academy of Sciences, Shanghai, China) were grown in DMEM supplemented with $10 \%$ FBS and penicillin/streptomycin $(100 \mathrm{U} / \mathrm{mL}$ and $100 \mu \mathrm{g} / \mathrm{mL}$, respectively; P1400, Solarbio, Beijing, China). Cells were cultured at $37^{\circ} \mathrm{C}$ in a humidified atmosphere of $5 \% \mathrm{CO}_{2}$.

Primary astrocytes were prepared as previously described with slight modification [20, 21]. In brief, cerebral cortices were dissected from brains of new-born pups of Institute of Cancer Research (ICR) mice (postnatal 1-2 days; purchased from the Experimental Animal Center of Wenzhou Medical University) in cold Hank's buffered saline. The animals used in this study were treated in accordance with protocols approved by the Institutional Animal Care and Use Committee of Wenzhou Medical University. Cortices were gently triturated and digested with $0.25 \%$ Trypsin-EDTA solution (25200056, Gibco, Grand Island, NY, USA) for $15 \mathrm{~min}$ at $37^{\circ} \mathrm{C}$. Thereafter, equal volume of culture medium, that is, DMEM/F-12 supplemented with $10 \%$ FBS and penicillin/streptomycin, was added and centrifuged at $200 \mathrm{~g}$ for $5 \mathrm{~min}$. The pellets were gently resuspended in culture medium and filtered through a $100 \mu \mathrm{m}$ pore mesh. The cells were then seeded in $75 \mathrm{~cm}^{2}$ flasks coated with poly-L-lysine $(1 \mathrm{mg} / \mathrm{mL})$ and cultured at $37^{\circ} \mathrm{C}$ with medium changed every 4 days. When cells reached a confluency of $90-95 \%$, the flasks were placed on an orbital shaker at $250 \mathrm{rpm}$ and $37^{\circ} \mathrm{C}$ for $16 \mathrm{~h}$. The remaining attached cells were then collected and re-cultured. Purity of astrocytes was evaluated by immunostaining of glial fibrillary acidic protein (GFAP). GFAP positive cells were counted in 3-random fields each time. Cultures were used for subsequent experiments when GFAP positivity was over $95 \%$ (Figure $1 \mathrm{~A}$ ).

\section{Immunofluorescence}

Cells were fixed in $4 \%$ paraformaldehyde for $30 \mathrm{~min}$, followed by permeabilization in $0.2 \%$ Triton X-100 for $15 \mathrm{~min}$ and then blocked with $5 \%$ bovine serum albumin (Beyotime, Shanghai, China) for $1 \mathrm{~h}$ at room 
temperature. The washing buffer is phosphate-buffered saline. Primary antibodies against GFAP and p65 were from Millipore (MAB360, Billerica, MA, USA) and Cell Signaling (8242S, Boston, MA, USA), respectively. Alexa Fluor 488-conjugated anti-mouse (A11001) and 555-conjugated anti-rabbit (A21428) IgG antibodies and Hoechst 33258 (H3569) were from Thermo Fisher (Rockford, IL, USA).

\section{Treatments and preparation of conditioned media}

Microglia conditioned media were prepared as previously [11]. BV2 cells were seeded at a density of 2-3 $\times$ $10^{6}$ in a $10-\mathrm{cm}$ culture plate and cultured for $24 \mathrm{~h}$ followed by serum starvation overnight. Cells were then treated with or without LPS (100 ng/mL; L4391, Sigma, St. Louis, MO, USA) for $2 \mathrm{~h}$, and then refreshed with serum-free medium with a continued incubation for $24 \mathrm{~h}$. Following centrifugation at $20,000 \mathrm{~g}$ for 5 min, the media were collected as microglia conditioned medium without LPS stimulation (M/C) or microglia conditioned medium pre-activated with LPS (M/Lps), and stored at $-80^{\circ} \mathrm{C}$ till use (Figure 1B).

Primary astrocytes of passage 3-5 were seeded at a density of 2-3 $\times 10^{6}$ per well in a 6-well plate and cultured for 24h. Following starvation overnight, cells were pretreated with paroxetine (P9623, Sigma, St. Louis, MO, USA) at indicated concentrations for $30 \mathrm{~min}$, followed by treatments of LPS (100 ng/mL) or the above $M / C$ and $M / L p s$ for indicated time. The resulted media of astrocytes treated with $M / C, M / L p s$ or LPS for $24 \mathrm{~h}$ were centrifuged at $20,000 \mathrm{~g}$ for $5 \mathrm{~min}$ and designated as A/MC (conditioned medium of astrocytes cultured with M/C), A/MLps (conditioned medium of astrocytes cultured with M/Lps) and A/Lps (conditioned medium of astrocytes cultured with LPS), respectively (Figure 1B). Media were stored at $-80^{\circ} \mathrm{C}$ till use.

\section{Cell viability measurement}

Cell viability was determined by the tetrazolium salt 3-[4,5-dimethylthiazol-2-yl] -2,5-diphenyltetrazolium bromide (MTT; C0009, Beyotime, Shanghai, China) assay [22]. Primary astrocytes or SH-SY5Y cells were seeded in triplicates in 96-well plates at $5 \times 10^{4}$ and $1 \times 10^{4}$ per well, respectively, and cultured for $24 \mathrm{~h}$. Astrocytes were treated with paroxetine at different concentrations for $24 \mathrm{~h}$ following overnight starvation. SH-SY5Y cells were incubated with A/MC, A/MLps, A/Lps or LPS directly $(100 \mathrm{ng} / \mathrm{mL})$ for 24 h. Thereafter, MTT ( $5 \mathrm{mg} / \mathrm{mL}$ prepared in phosphate-buffered saline) was added into each well and incubated at $37^{\circ} \mathrm{C}$ for $4 \mathrm{~h}$. The resulting formazan crystals were dissolved in dimethyl sulfoxide. Optical density was measured at $490 \mathrm{~nm}$.

\section{RNA isolation and quantitative PCR}

Total RNA was isolated using TRIzol Reagent (15596018, Invitrogen, Carlsbad, CA, USA), and reversetranscribed to cDNA using PrimeScript RT kit (RR037A, TaKaRa, Dalian, China). Quantitative PCR was performed using SYBR Green Supermix (1725260, Bio-Rad, Hercules, CA, USA) following the manufacturer's instruction. Primers were as follows: TNF-a, 5'-CGT CAG CCG ATT TGC TAT CT-3' and 5'CGG ACT CCG CAA AGT CTA AG-3'; IL-1ß, 5'-GAA ATG CCA CCT TTT GAC AGT G-3' and 5'-TGG ATG CTC TCA TCA GGA CAG-3'; BDNF, 5'-TCA TAC TTC GGT TGC ATG AAG G-3' and 5'-AGA CCT CTC GAA CCT GCC 
C-3'; MANF, 5'-TCT GGG ACG ATT TTA CCA GGA-3' and 5'-CTT GCT TCA CGG CAA AAC TTT-3'; $\beta$-actin, 5'AGC CAT GTA CGT AGC CAT CC-3' and 5'-CTC TCA GCT GTG GTG GTG AA-3'.

\section{Determination of TNF- $a$ and BDNF production}

Medium TNF- $a$ and BDNF were measured using ELISA kits respectively from R\&D Systems (VAL609, Minneapolis, MN, USA) and Westang (F10200, Shanghai, China) according to the manufacturers' instructions. Absorbance was read at $450 \mathrm{~nm}$. The concentration of each sample was calculated from the standard curve prepared using the included standards.

\section{NO production assay}

Medium nitrite was measured as an indicator of NO production [23]. In brief, $50 \mu \mathrm{l}$ of supernatant was mixed with an equal volume of Griess reagent I, followed by an addition of $50 \mu$ l of Griess reagent II (S0021, Beyotime, Shanghai, China) at room temperature. Absorbance was immediately measured at 540 $\mathrm{nm}$. The concentration of each sample was calculated from a standard curve generated using sodium nitrite.

\section{Western blot analysis}

Cells were lysed in sample buffer containing $60 \mathrm{mM}$ Tri-HCl, pH 6.8, 2\% SDS and 5\% glycerol, and boiled for 5 min. Total protein concentration was measured using a BCA kit (P0010, Beyotime, Shanghai, China). Equal amount of protein from each sample was loaded and analyzed by Western blot as previously described [24]. Pyrrolidine dithiocarbamic acid (PDTC; P8765) was purchased from Sigma (St. Louis, MO, USA). Primary antibodies against p-p65 (3031), p65 (3034), p-JNK1/2 (9251), JNK1/2 (9252), p-p38 (9211), p38 (9212), p-ERK1/2 (9101), ERK1/2 (9102), p-STAT3 (9145), STAT3 (12640), iNOS (2977) and $\beta$-actin (4970), anti-rabbit (7074) and anti-mouse (7076) secondary antibodies, and LumiGLO ${ }^{8}$ Reagent and Peroxide chemiluminescence detection kit (7003) were all purchased from Cell Signaling (Boston, MA, USA).

\section{Statistical analysis}

Data were analyzed by one-way analysis of variance (ANOVA) followed by the Tukey's post hoc test for multiple comparisons and two-way ANOVA for factorial design experiments using the SPSS 23.0 statistics software. Values were expressed as mean \pm SE from at least three independent experiments. The $p<0.05$ was considered statistically significant.

\section{Results}

\section{Effect of paroxetine on LPS-induced inflammatory responses in primary astrocytes}

To understand potential toxicity of paroxetine on primary astrocytes, we treated cells with paroxetine at 0 , $1,3,5,10$ and $20 \mu \mathrm{M}$ for $24 \mathrm{~h}$. Results showed that paroxetine at $20 \mu \mathrm{M}$, but not at the other doses, 
induced significant cell death [Figure $2 ; F_{(5,12)}=15.38, p<0.001$; post hoc, $p=0.001(0 \mu \mathrm{M}$ vs $20 \mu \mathrm{M})$ ].

LPS stimulated light iNOS expression in isolated primary astrocytes [Figure $3 A ; F_{(6,14)}=31.82, p<0.001$; full blots listed in Figure S1], as the induction is much weaker than that in microglia following the same treatment [19]. As a consequence, no significant elevation in NO levels was detected in astrocytes upon LPS treatment (Figure 3B). LPS induced significant increase in TNF-a and IL-1 $\beta$ mRNA expression [Figure

3C; TNF-a: $F_{(6,21)}=56.12, p<0.001$; IL-1 $\beta: F_{(6,21)}=67.68, p<0.001$ ] and extracellular release of TNF- $\mathrm{a}$ [Figure 3D; $\left.F_{(6,14)}=225.56, p<0.001\right]$. However, paroxetine pretreatments at different concentrations did not lead to apparent changes in iNOS expression and NO production (Figures $3 \mathrm{~A}$ and B), neither in TNF-a and IL-1 $\beta$ mRNA expression and TNF-a production (Figures 3C and D).

\section{Effect of paroxetine on M/Lps-induced inflammatory responses in primary astrocytes}

The iNOS expression upon M/Lps incubation was barely detectable (data not shown). To determine whether there was an increase in production of cytokines derived from astrocytes, the NO and TNF-a carried from $\mathrm{M} / \mathrm{C}$ or $\mathrm{M} / \mathrm{Lps}$ per se (without treating astrocytes) were measured being their baseline levels (Figure 4A and $\mathrm{C}$; the right last two columns). There was no significant production of NO by astrocytes treated with M/Lps compared to its baseline level (Figure 4A). M/Lps induced significant increase in TNF$a$ and IL-1 $\beta$ mRNA expression in primary astrocytes. The induction was suppressed by pretreatments of paroxetine, in particular at doses of 5 and $10 \mu \mathrm{M}$ [Figure 4B; TNF-a: $F_{(6,21)}=14.77, p<0.001$; post hoc, $p=$ 0.037 (M/Lps vs M/Lps + paroxetine $10 \mu \mathrm{M}$; down by 42.2\%); IL-1 $\beta: F_{(6,21)}=15.88, p<0.001$; post hoc, $p$ $=0.027(\mathrm{M} / \mathrm{Lps}$ vs $\mathrm{M} / \mathrm{Lps}+$ paroxetine $5 \mu \mathrm{M}$; down by $46.7 \%), p=0.002(\mathrm{M} / \mathrm{Lps} v s \mathrm{M} / \mathrm{Lps}+$ paroxetine $10 \mu \mathrm{M}$; down by $61.8 \%)]$. Although not significant, a slight increase appeared in the production of TNF-a in media of astrocytes incubated with M/Lps compared to its baseline level (the third vs the ninth). This increase was blunted by pretreatments of paroxetine at doses of 5 and $10 \mu \mathrm{M}$ [Figure 4C; $F_{(8,27)}=99.06, p$ $<0.001$; post hoc, $p=0.013$ (M/Lps vs M/Lps + paroxetine $5 \mu \mathrm{M}$; down by $21.9 \%), p=0.011$ (M/Lps vs $\mathrm{M} /$ Lps + paroxetine $10 \mu \mathrm{M}$; down by $22.3 \%)]$.

\section{Paroxetine inhibits M/Lps-induced inflammatory responses in primary astrocytes partially through NF-KB pathway}

To understand the underlying mechanism of paroxetine inhibition on M/Lps-induced cytokine expression, we analyzed the mitogen-activated protein kinase (MAPK), NF-KB and STAT3 signaling pathways. Cells were pretreated with paroxetine for 30 min followed by treatment of M/Lps for the indicated time. As shown in the comparisons of 0 min (Figure 5), paroxetine alone had little impact on the activation of these proteins. The p38, JNK1/2, ERK1/2, p65/NF-KB and STAT3 were all markedly activated in primary astrocytes upon $\mathrm{M} / \mathrm{Lps}$ treatments (Figure $5 \mathrm{~A}$ ). However, the phosphorylation of p38, JNK1/2, ERK1/2, and STAT3 was not suppressed by paroxetine pretreatment except that of p65/NF-kB (Figure 5B-F). The inhibition rate for p-p65 was 33.7, 40.2, 49.4 and 53.6\%, respectively, at 15, 30, 60 and 120 min [Figure 5E; M/Lps*time interaction: $F_{(4,30)}=3.08, p=0.031 ; \mathrm{M} / \mathrm{Lps}+$ paroxetine $v s \mathrm{M} / \mathrm{Lps}: p=0.001$ (15 min), $p=$ 0.004 (30 min), $p<0.001$ (60 min), $p=0.001$ (120 min)]. Immunofluorescence further showed that M/Lps 
stimulated nuclear translocation of $\mathrm{p} 65$ and this translocation was blocked by paroxetine pretreatment [Figure 6; time* paroxetine interaction: $F_{(1,8)}=12.30, p=0.008$; M/Lps + paroxetine vs M/Lps: $p=0.012$ (30 min)]. Pretreatment of PDTC (a specific inhibitor of NF-KB pathway; Figure 7A; M/Lps*PDTC interaction: $F_{(1,8)}=27.24, p=0.001 ; \mathrm{M} / \mathrm{Lps}+$ PDTC $\left.v s \mathrm{M} / \mathrm{Lps}: p<0.001\right)$ reduced the M/Lps-induced elevation of TNF- $\alpha$ and IL-1 $\beta$ mRNA expression by $18.0 \%$ and $39.0 \%$, respectively in the astrocytes (Figure 7B; TNF-a: $F_{(1,8)}=22.14, p=0.002 ; \mathrm{M} / \mathrm{Lps}+$ PDTC vs M/Lps: $p<0.001 ; \mathrm{IL}-1 \beta: F_{(1,8)}=18.73, p=0.003$; M/Lps + PDTC vs M/Lps: $p<0.001)$.

We additionally examined the effect of paroxetine on p65/NF-kB activation in the context of LPS stimulation. Results showed that LPS induced little p65 phosphorylation (data not shown) but stimulated apparent p65 nuclear translocation $(p<0.05)$. However, in concord with its effect on cytokine production, paroxetine did not blunt this translocation (Figure S2).

\section{A/MLps with paroxetine has little impact on SH-SY5Y cell viability}

To investigated whether neuronal survival was affected by the reactive-microglia mediated activation of astrocytes, LPS-activated astrocytes and LPS per se, we cultured SH-SY5Y cells, a neuroblastoma cell line commonly used for PD study, with A/MLps, A/Lps and LPS. Results showed that A/MLps, A/Lps and LPS regardless of paroxetine pretreatment resulted in little impact on the survival of SH-SY5Y cells (Figure 8).

We further investigated whether potential neurotrophic support of the astrocytes played a role amidst. The mRNA expression of neurotrophic factors, in particular BDNF, was increased in astrocytes following the incubation with M/Lps [Figure 9A; BDNF: $F_{(6,14)}=16.41, p<0.001$; post hoc, $p=0.012$ (M/C vs M/Lps; up by $89 \%)$; MANF: $\left.F_{(6,14)}=5.06, p=0.006\right]$. In striking contrast to the results in cytokines, paroxetine did not suppress the M/Lps-induced expression of these neurotrophic factors. Instead, paroxetine at $10 \mu \mathrm{M}$ led to higher mRNA expression of BDNF and MANF in astrocytes [BDNF: post hoc, $p<0.001$ (M/C vs M/C + paroxetine $10 \mu \mathrm{M}$; up by 152\%), $p=0.003$ (M/Lps vs M/Lps + paroxetine 10 $\mu \mathrm{M}$; up by 56\%); MANF: $p=$ 0.037 (M/C vs M/C + paroxetine $10 \mu \mathrm{M}$; up by $88 \%)$ ]. Similarly, paroxetine at $10 \mu \mathrm{M}$ resulted in a trend of elevation in medium BDNF levels compared with their respective controls (Figure 9B). BDNF concentration was significantly increased in media of the astrocytes incubated with $\mathrm{M} / \mathrm{Lps}$ compared to the M/C incubation $\left(F_{(5,18)}=15.61, p<0.001\right.$; post hoc, $\left.p=0.003\right)$. Interestingly, compared to the baseline levels in the conditioned media, medium BNDF levels appeared to be reduced upon incubation with the astrocytes (Figure 9B; the $1^{\text {st }}$ vs $5^{\text {th }}$ column, the $3^{\text {rd }}$ vs $6^{\text {th }}$ column).

\section{Discussion}

Neuroinflammation is involved in the pathophysiology of neurodegenerative diseases such as PD and Alzheimer's disease [1-3]. Targeting microglia- and astrocyte-mediated immune responses is therapeutically promising against these disorders [25]. Besides being a drug against depression, paroxetine is shown to inhibit MPTP-induced loss of nigrostriatal dopaminergic neurons and neuroinflammatory responses, and to suppress LPS-induced microglia activation $[16,19,26]$. Results of 
the current study in primary astrocytes suggest that 1) paroxetine inhibits reactive microglia-mediated, but not LPS-induced, astrocytic inflammatory responses; 2) LPS-induced microglia activation dominates over its subsequent activation of astrocytes; 3 ) paroxetine-mediated astrocytic responses during LPS insult has little actual impact on neuronal survival.

Astrocytes are often activated following activation of microglia, which may stimulate astrocytes in a paracrine pathway [27]. Incubation of astrocytes with microglia conditioned medium indicates a paracrine regulation of microglia on astrocytic responses. Indeed, reactive microglia stimulate mRNA expression of both cytokines such as TNF- $\alpha$ and IL-1 $\beta$ and neurotrophic factors such as BDNF and MANF in astrocytes. However, compared to the reactive microglia per se, this paracrine activation of astrocytes may have a rather small effect on surroundings as suggested by the light extracellular release of effectors in media. It is not surprising given that microglia are presumably more potent than astrocytes leading to neuroinflammation $[4,5]$. In consistency, the induction of iNOS by LPS in astrocytes is considerably less than that in microglia [19], which may explain why the NO production is not significantly elevated in astrocytes. Sheng et al. also showed that NO production was not detectable in either immortalized rat astrocytes or primary astrocytes upon treatment of LPS at $100 \mathrm{ng} / \mathrm{mL}$ [28].

However, while reactive microglia indeed release neurotrophic factors [30,31], we initially thought that astrocytes should have been more potent secreting neurotrophic factors upon being activated. Unexpectedly, there is no drastic increase of BDNF in the astrocyte media compared to its baseline levels of microglia conditioned media. Instead, astrocytes seem to absorb microglia-released neurotrophic factors as observed. On the other hand, paroxetine indeed upregulates mRNA expression and extracellular release of neurotrophic factors such as BDNF as being previously noted [29].

Saijo et al. reported that microglia-mediated inflammatory responses were magnified by astrocytes leading to more neuronal death [11]. However, as above discussed, this magnification is not displayed in the current study. Both studies used primary astrocytes and BV2 cells for preparing conditioned media, whereas the latter is considered as a valid substitute for primary microglia in complex cell-cell interaction studies [32]. In an effort to interpret the above discrepancy, we observed that their BV2 cells used to make conditioned media were all infected with lentivirus [11]. As manifested in one of their results, LPSactivated conditioned media from this type of cells exhibited significantly higher toxicity to tyrosine hydroxylase positive neurons compared to those prepared from un-infected cells, suggesting that microglia upon lentivirus infection are more reactive and potent generating pro-inflammatory cytokines. Consequently, paracrine factors in their microglia conditioned media are presumably of much higher concentrations, then leading to more drastic astrocytic responses than in the current study.

Paroxetine prevents LPS-induced inflammatory responses in microglia [19], but not in astrocytes. Toll-like receptor distribution varies considerably between microglia and astrocytes [33]. For instance, TLR4, the major LPS receptor, is expressed extensively in microglia, but this receptor and its downstream signaling component MYD88 are deficient in rodent astrocytes [34,35]. Thus, the signaling pathways in response to LPS much differ in these two types of immune cells. Paroxetine markedly suppresses the reactive 
microglia-induced activation of p65/NF-kB in astrocytes including both phosphorylation and nuclear translocation. Interestingly, the nuclear translocation of $\mathrm{p} 65$ induced by a direct LPS treatment was not blunted by paroxetine. These results suggest that the two kinds of stimuli, LPS and cytomix from conditioned media, have different intermediate effectors in astrocytes. Completely blocking NF-KB pathway by PDTC results in less inhibition in cytokine expression compared to those by paroxetine ( $18.0 \%$ vs $42.2 \%$, and $39.0 \%$ vs. $61.8 \%$, respectively for the reductions in TNF-a and IL $-1 \beta$ expression). Thus, additional pathways should be involved in the paroxetine suppression of astrocytic inflammatory responses. STAT3 and MAPKs including p38, JNK and ERK are considered to be key regulators in astrocytes in response to neuroinflammation besides NF-KB [36]. However, it turns out that both MAPKs and STAT3 are not the answer.

We have previously reported that incubation with LPS-stimulated microglia conditioned media leads to a significant death of SH-SY $5 Y$ cells by about $15 \%$, where for example the medium TNF-a level is at approximately $8,500 \mathrm{pg} / \mathrm{mL}$ [19]. No significant impact on SH-SY5Y cell viability is observed when incubated with the reactive microglia- or LPS-stimulated astrocyte conditioned media, where the level of TNF-a is roughly only at 1,100 and $100 \mathrm{pg} / \mathrm{mL}$, respectively as a comparison. Minor amounts of cytokines and neurotrophic factors are derived from astrocytes as above mentioned. Paroxetine thus renders little impact on the neuronal survival under this condition.

\section{Conclusions}

The current study demonstrates that paroxetine ameliorates the reactive microglia-mediated inflammatory responses in astrocytes partially via inhibition of NF-KB pathway, but has no impact on LPS-stimulated astrocyte activation. Paroxetine also stimulates neurotrophic support of astrocytes. While this secondary astrocytic response appears minor compared to the innate immune responses of microglia, paroxetine can indeed alleviate neuroinflammation by suppressing microglia activation [19] and its paracrine inflammatory activation of astrocytes. Our results support a therapeutic potential of paroxetine against neuroinflammation-associated neurological disorders such as PD.

\section{Abbreviations}

A/Lps, conditioned medium of astrocytes cultured with LPS; A/MC, conditioned medium of astrocytes cultured with $M / C ; A / M L p s$, conditioned medium of astrocytes cultured with $M / L p s ; B D N F$, brain derived neurotrophic factor; ERK, extracellular signal-regulated kinase; GFAP, glial fibrillary acidic protein; IL-1 $1 \beta$, interleukin 1 $\beta$; iNOS, inducible nitric oxide synthase; JNK, c-jun N-terminal kinase; LPS, lipopolysaccharide; MANF, mesencephalic astrocyte-derived neurotrophic factor; MAPK, mitogen-activated protein kinase; $\mathrm{M} / \mathrm{C}$, microglia conditioned medium without LPS stimulation; $M / L p s$, microglia conditioned medium preactivated with LPS; NF-kB, nuclear factor KB; NO, nitric oxide; PD, Parkinson's disease; PDTC, pyrrolidine dithiocarbamic acid; STAT3, signal transducer and activator of transcription 3; TLR, Toll-like receptor; TNF-a, tumor necrosis factor $a$. 


\section{Declarations}

\section{Ethics approval and consent to participate}

The animals used in this study were treated in accordance with protocols approved by the Institutional Animal Care and Use Committee of Wenzhou Medical University, China.

\section{Consent for publication}

Not applicable

\section{Availability of data and material}

All data supporting the conclusions of this study are included within the article.

\section{Competing interests}

The authors declare that there is no potential conflict of interest.

\section{Funding}

The study was supported by funding from Zhejiang Provincial Natural Science Foundation (LD19H090001, and LZ19H090002), National Natural Science Foundation of China (81571087, 81771380, and 81771510), Wenzhou Municipal Science and Technology Bureau (Y20170071 and C20170003).

\section{Authors' contributions}

$X Z$, JHZ and CPH designed the study, LBZ, JHH, HQZ, SYJ, CNZ, and NNH conducted the experiments, XZ, $\mathrm{JHH}, \mathrm{LBZ}$, and $\mathrm{JHZ}$ analyzed the data, $\mathrm{JHZ}, \mathrm{CPH}$ and $\mathrm{JHH}$ wrote the manuscript. All authors read and approved the final manuscript.

\section{Acknowledgements}

Not applicable. 


\section{References}

1.Poewe W, Seppi K, Tanner C, Halliday G, Brundin P, Volkmann J, et al. Parkinson disease. Nat. Rev. Dis. Primers. 2017;3:17013.

2.Ransohoff R. How neuroinflammation contributes to neurodegeneration. Science. 2016;353:777-83.

3.Sliter DA, Martinez J, Hao L, Chen X, Sun N, Fischer TD, et al. Parkin and PINK1 mitigate STING-induced inflammation. Nature. 2018;561:258-262.

4.Halliday G, Stevens C. Glia: initiators and progressors of pathology in Parkinson's disease. Mov. Disord. 2011;26:6-17.

5.Shastri A, Bonifati DM, Kishore U. Innate immunity and neuroinflammation. Mediators Inflamm. 2013;2013:342931.

6.Croisier E, Moran LB, Dexter DT, Pearce RK, Graeber MB. Microglial inflammation in the parkinsonian substantia nigra: relationship to alpha-synuclein deposition. J Neuroinflammation. 2005;2:14.

7.Duffy M, Collier T, Patterson J, Kemp C, Luk K, Tansey M, et al. Lewy body-like alpha-synuclein inclusions trigger reactive microgliosis prior to nigral degeneration. J. Neuroinflammation. 2018;15:129.

8.Booth HDE, Hirst WD, Wade-Martins R. The Role of Astrocyte Dysfunction in Parkinson's Disease Pathogenesis. Trends Neurosci. 2017;40:358-370.

9.Miklossy J, Doudet D, Schwab C, Yu S, McGeer E, McGeer P. Role of ICAM-1 in persisting inflammation in Parkinson disease and MPTP monkeys. Exp. Neurol. 2006;197:275-83.

10.Sofroniew M, Vinters H. Astrocytes: biology and pathology. Acta Neuropathol. 2010;119:7-35.

11.Saijo K, Winner B, Carson CT, Collier JG, Boyer L, Rosenfeld MG, et al. A Nurr1/CoREST pathway in microglia and astrocytes protects dopaminergic neurons from inflammation-induced death. Cell. 2009;137:47-59.

12.Hwang J, Zheng L, Ock J, Lee M, Kim S, Lee H, et al. Inhibition of glial inflammatory activation and neurotoxicity by tricyclic antidepressants. Neuropharmacology. 2008;55:826-34.

13.Mansouri M, Naghizadeh B, Ghorbanzadeh B, Alboghobeish S, Amirgholami N, Houshmand G, et al. Venlafaxine prevents morphine antinociceptive tolerance: The role of neuroinflammation and the Iarginine-nitric oxide pathway. Exp. Neurol. 2018;303:134-141.

14.Vollmar P, Haghikia A, Dermietzel R, Faustmann PM. Venlafaxine exhibits an anti-inflammatory effect in an inflammatory co-culture model. Int. J. Neuropsychopharmacol. 2008;11:111-7. 
15.Zhao Y, Pan Y, Tang M, Lin W. Blocking p38 Signaling Reduces the Activation of Pro-inflammatory Cytokines and the Phosphorylation of p38 in the Habenula and Reverses Depressive-Like Behaviors Induced by Neuroinflammation. Front Pharmacol. 2018;9:511.

16.Chung Y, Kim S, Jin B. Paroxetine prevents loss of nigrostriatal dopaminergic neurons by inhibiting brain inflammation and oxidative stress in an experimental model of Parkinson's disease. J. Immunol. 2010;185:1230-7.

17.Meredith GE, Sonsalla PK, Chesselet MF. Animal models of Parkinson's disease progression. Acta Neuropathol. 2008;115:385-98.

18.Gao H, Zhang F, Zhou H, Kam W, Wilson B, Hong J. Neuroinflammation and a-synuclein dysfunction potentiate each other, driving chronic progression of neurodegeneration in a mouse model of Parkinson's disease. Environ. Health Perspect. 2011;119:807-14.

19.Liu RP, Zou M, Wang JY, Zhu JJ, Lai JM, Zhou LL, et al. Paroxetine ameliorates lipopolysaccharideinduced microglia activation via differential regulation of MAPK signaling. J. Neuroinflammation. 2014;11:47.

20.McCarthy KD. Preparation of separate astroglial and oligodendroglial cell cultures from rat cerebral tissue. J. Cell Biol. 1980;85:890-902.

21.Shen Y, Qin H, Chen J, Mou L, He Y, Yan Y, et al. Postnatal activation of TLR4 in astrocytes promotes excitatory synaptogenesis in hippocampal neurons. J. Cell Biol. 2016;215:719-734.

22.Zhu JH, Lei XG. Double null of selenium-glutathione peroxidase-1 and copper, zinc-superoxide dismutase enhances resistance of mouse primary hepatocytes to acetaminophen toxicity. Exp. Biol. Med. (Maywood). 2006;231:545-52.

23.Wilms H, Sievers J, Rickert U, Rostami-Yazdi M, Mrowietz U, Lucius R. Dimethylfumarate inhibits microglial and astrocytic inflammation by suppressing the synthesis of nitric oxide, IL-1beta, TNF-alpha and IL-6 in an in-vitro model of brain inflammation. J. Neuroinflammation. 2010;7:30.

24.Zhu JH, Chen CL, Flavahan S, Harr J, Su B, Flavahan NA. Cyclic stretch stimulates vascular smooth muscle cell alignment by redox-dependent activation of Notch3. Am. J. Physiol. Heart Circ. Physiol. 2011;300:H1770-80.

25.Wang Q, Liu Y, Zhou J. Neuroinflammation in Parkinson's disease and its potential as therapeutic target. Transl. Neurodegener. 2015;4:19.

26.Tynan RJ, Weidenhofer J, Hinwood M, Cairns MJ, Day TA, Walker FR. A comparative examination of the anti-inflammatory effects of SSRI and SNRI antidepressants on LPS stimulated microglia. Brain Behav. Immun. 2012;26:469-79. 
27.Glass CK, Saijo K, Winner B, Marchetto MC, Gage FH. Mechanisms underlying inflammation in neurodegeneration. Cell. 2010;140:918-34.

28.Sheng W, Zong Y, Mohammad A, Ajit D, Cui J, Han D, et al. Pro-inflammatory cytokines and lipopolysaccharide induce changes in cell morphology, and upregulation of ERK1/2, iNOS and SPLAX-IIA expression in astrocytes and microglia. J. Neuroinflammation. 2011;8:121.

29.Allaman I, Fiumelli $H$, Magistretti P, Martin J. Fluoxetine regulates the expression of neurotrophic/growth factors and glucose metabolism in astrocytes. Psychopharmacology (Berl.). 2011;216:75-84.

30.Trang T, Beggs S, Wan X, Salter M. P2X4-receptor-mediated synthesis and release of brain-derived neurotrophic factor in microglia is dependent on calcium and p38-mitogen-activated protein kinase activation. J. Neurosci. 2009;29:3518-28.

31.Zhang X, Zeng L, Yu T, Xu Y, Pu S, Du D, et al. Positive feedback loop of autocrine BDNF from microglia causes prolonged microglia activation. Cell Physiol. Biochem. 2014;34:715-23.

32.Henn A, Lund S, Hedtjarn M, Schrattenholz A, Porzgen P, Leist M. The suitability of BV2 cells as alternative model system for primary microglia cultures or for animal experiments examining brain inflammation. Altex. 2009;26:83-94.

33.Bennett ML, Bennett FC, Liddelow SA, Ajami B, Zamanian JL, Fernhoff NB, et al. New tools for studying microglia in the mouse and human CNS. Proc. Natl. Acad. Sci. USA. 2016;113:E1738-46.

34.Cahoy JD, Emery B, Kaushal A, Foo LC, Zamanian JL, Christopherson KS, et al. A transcriptome database for astrocytes, neurons, and oligodendrocytes: a new resource for understanding brain development and function. J. Neurosci. 2008;28:264-78.

35.Zhang Y, Sloan SA, Clarke LE, Caneda C, Plaza CA, Blumenthal PD, et al. Purification and Characterization of Progenitor and Mature Human Astrocytes Reveals Transcriptional and Functional Differences with Mouse. Neuron. 2016;89:37-53.

36.Colombo E, Farina C. Astrocytes: Key Regulators of Neuroinflammation. Trends Immunol. 2016;37:608-620.

\section{Figures}




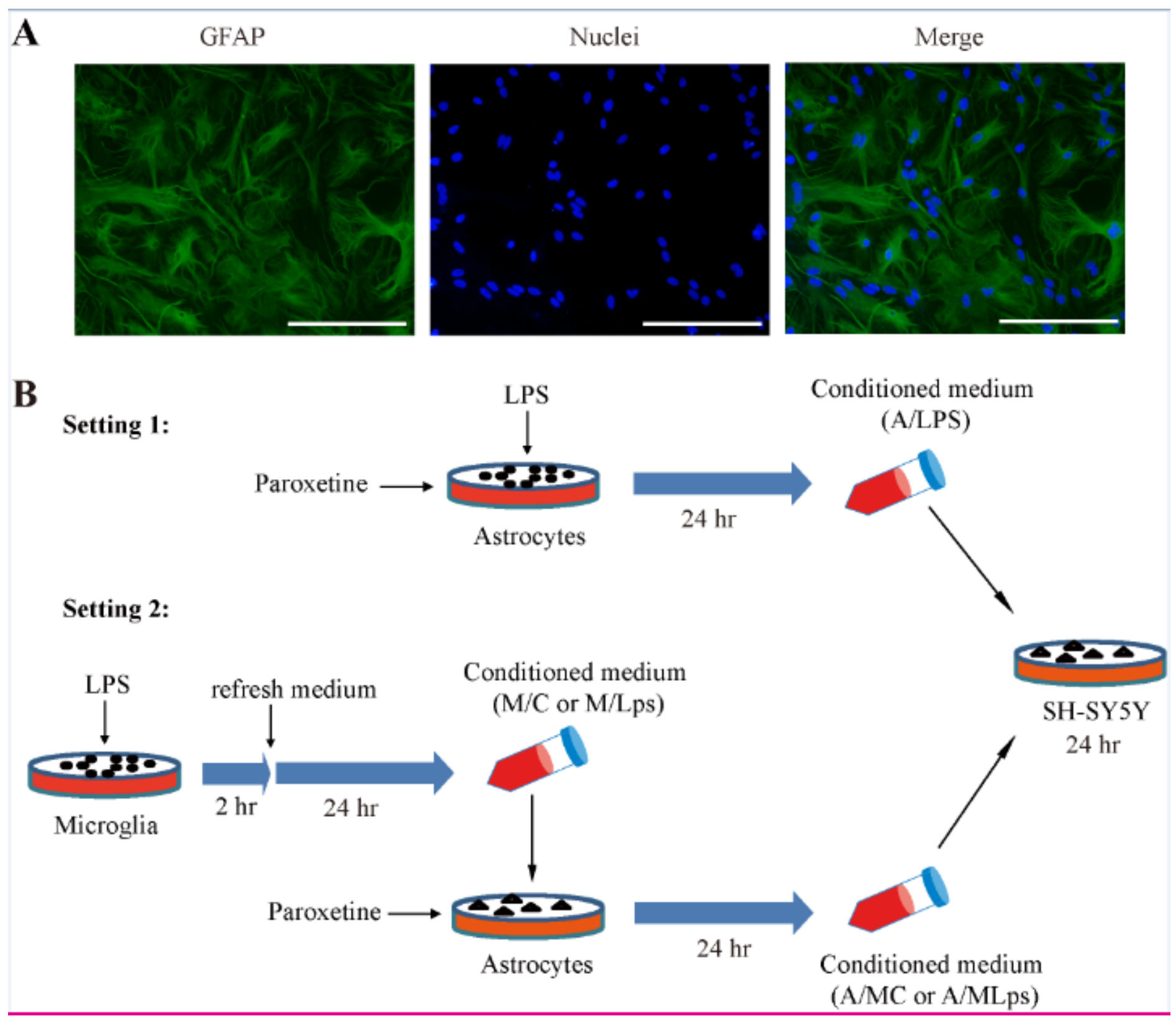

Figure 1

Isolation of primary astrocytes and experimental design. (A) Representative images of isolated primary astrocytes. Green, GFAP; Blue, nuclei; bar size, $100 \mu \mathrm{m}$. (B) Illustration of experimental design and conditioned media. A/Lps, conditioned medium of astrocytes cultured with LPS; A/MC, conditioned medium of astrocytes cultured with M/C; $A / M L p s$, conditioned medium of astrocytes cultured with M/Lps; GFAP, glial fibrillary acidic protein; LPS, lipopolysaccharide; M/C, microglia conditioned medium without LPS stimulation; M/Lps, microglia conditioned medium pre-activated with LPS. 


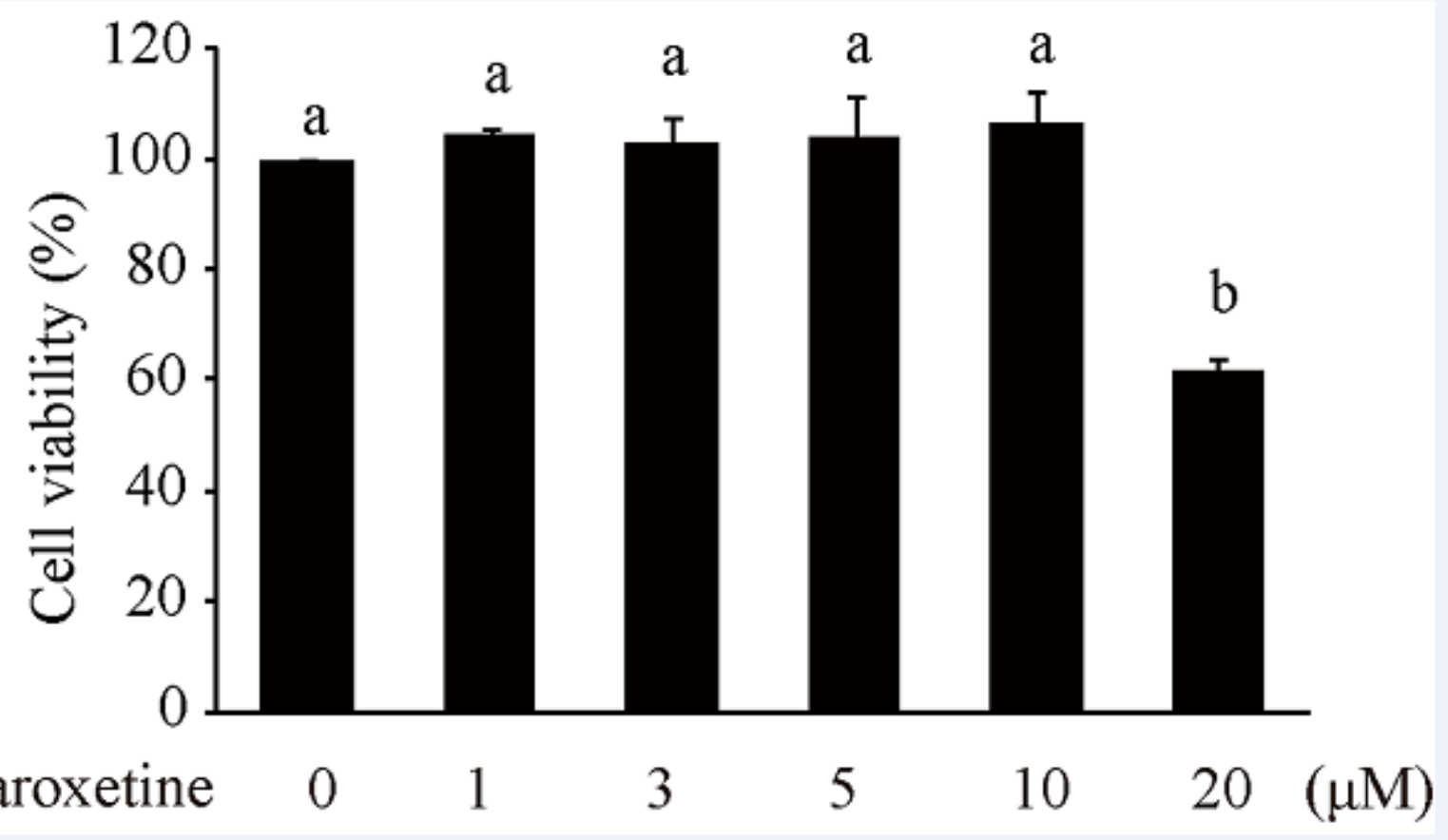

Figure 2

Impact of paroxetine on viability of primary astrocytes. Cells were treated with paroxetine at different concentrations for $24 \mathrm{~h}$. Cell viability was expressed as percentage of the control $(0 \mu \mathrm{M})$, which was set as $100 \%$. Values are means $\pm S E, n=3$. Statistical comparisons were performed using one-way ANOVA, followed by the Tukey's post hoc test. Different letters indicate $p<0.05$. 
$\mathbf{A}$
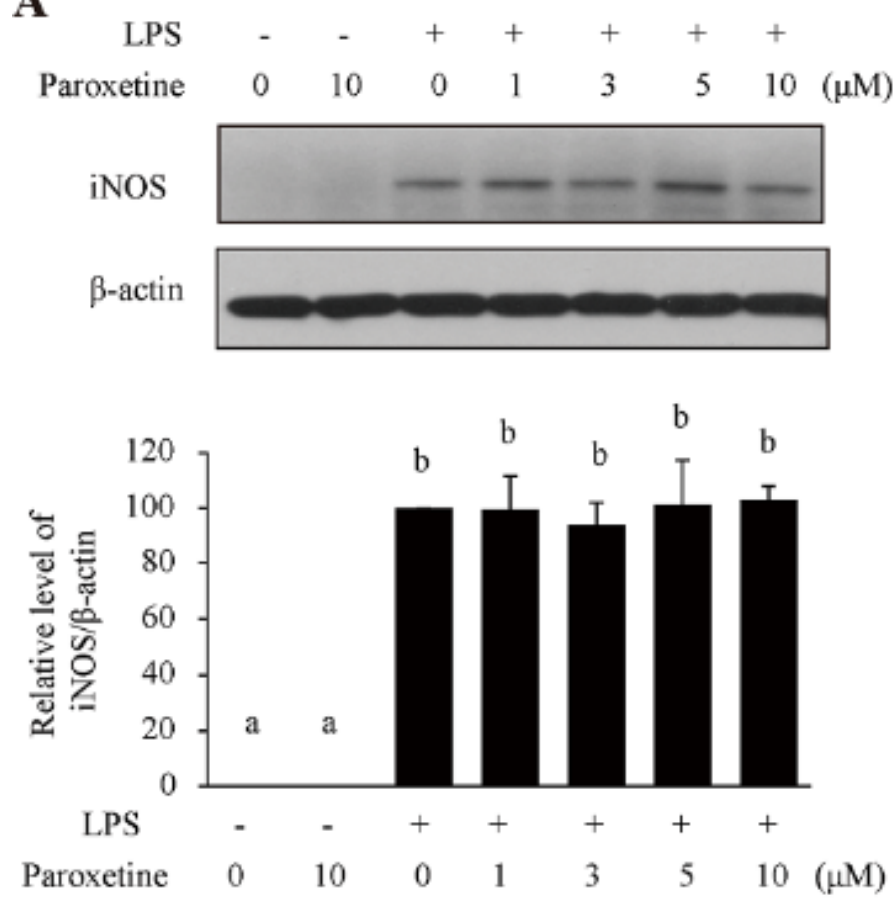

B

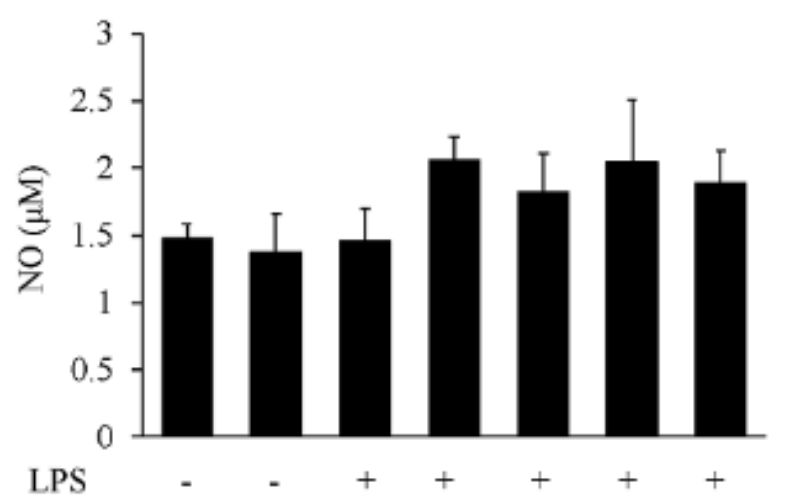

C
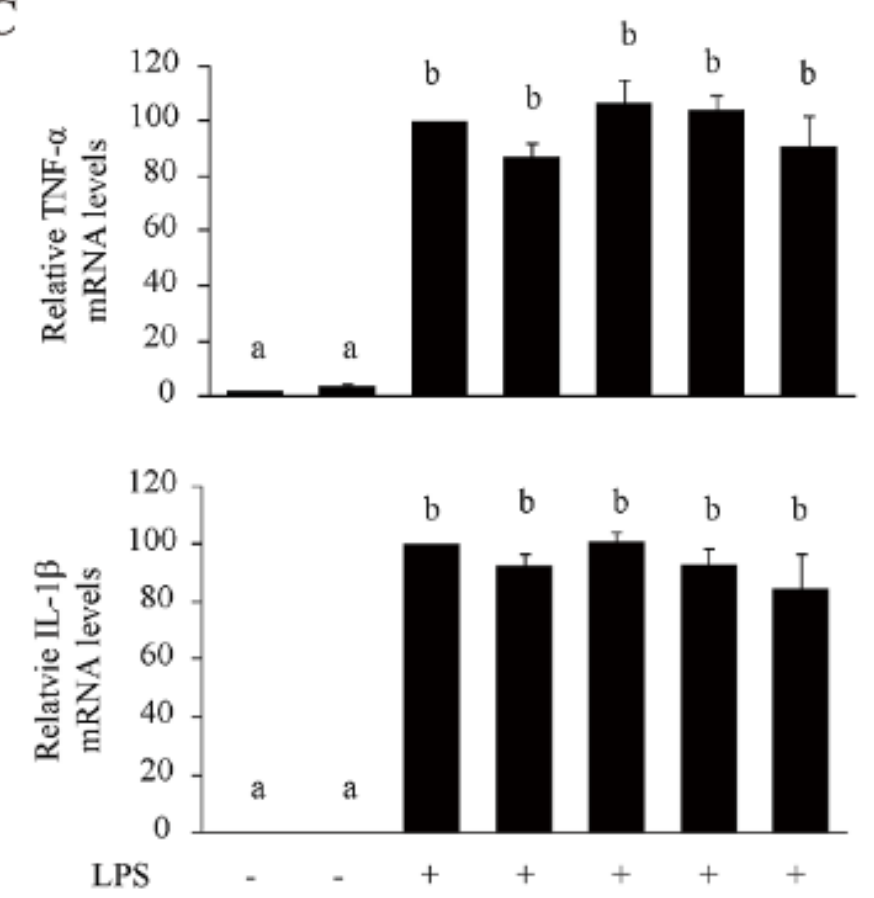

$\begin{array}{lllllllll}\text { Paroxetine } & 0 & 10 & 0 & 1 & 3 & 5 & 10 & (\mu \mathrm{M})\end{array}$

D

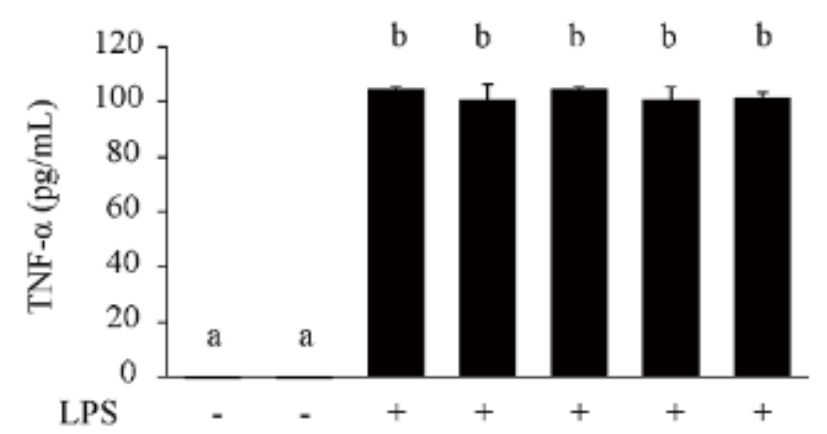

$\begin{array}{lllllllll}\text { Paroxetine } & 0 & 10 & 0 & 1 & 3 & 5 & 10 & (\mu \mathrm{M})\end{array}$

Paroxetine

$0 \quad 10$

$\begin{array}{llll}3 & 5 & 10 & (\mu \mathrm{M})\end{array}$

Figure 3

Effect of paroxetine on LPS-induced inflammatory responses in primary astrocytes. Cells were pretreated with paroxetine at different concentrations for 30 min followed by stimulation with LPS at $100 \mathrm{ng} / \mathrm{mL}$ for 24 h. (A) Western blot analysis of iNOS expression. Protein levels were quantified and normalized to their respective $\beta$-actin levels. Values were expressed relative to the one treated with LPS alone, which was set as 100. (B) NO production indicated by nitrite levels in culture media. (C) Quantitative PCR analyses of TNF- $\alpha$ and IL-1 $\beta$ expression. The mRNA levels were expressed relative to the one treated with LPS alone, which was set as 100. (D) Concentrations of TNF- $a$ in culture media. Values are means $\pm S E, n=3$ for (A, $B, D), n=4$ for (C). Statistical comparisons were performed using one-way ANOVA, followed by the Tukey's post hoc test. Different letters indicate $p<0.05$. IL-1 $\beta$, interleukin $1 \beta$; iNOS, inducible nitric oxide synthase; LPS, lipopolysaccharide; NO, nitric oxide; TNF-a, tumor necrosis factor a. 
A

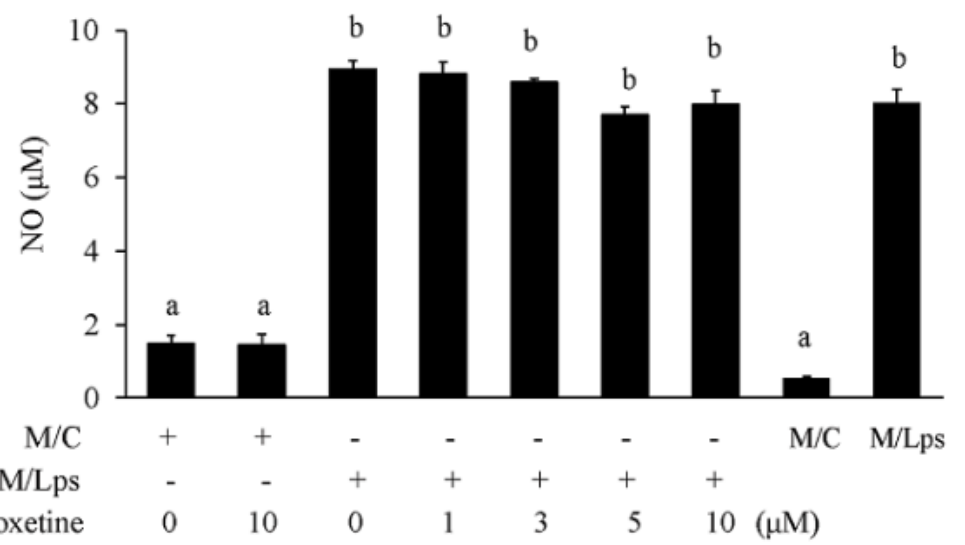

C

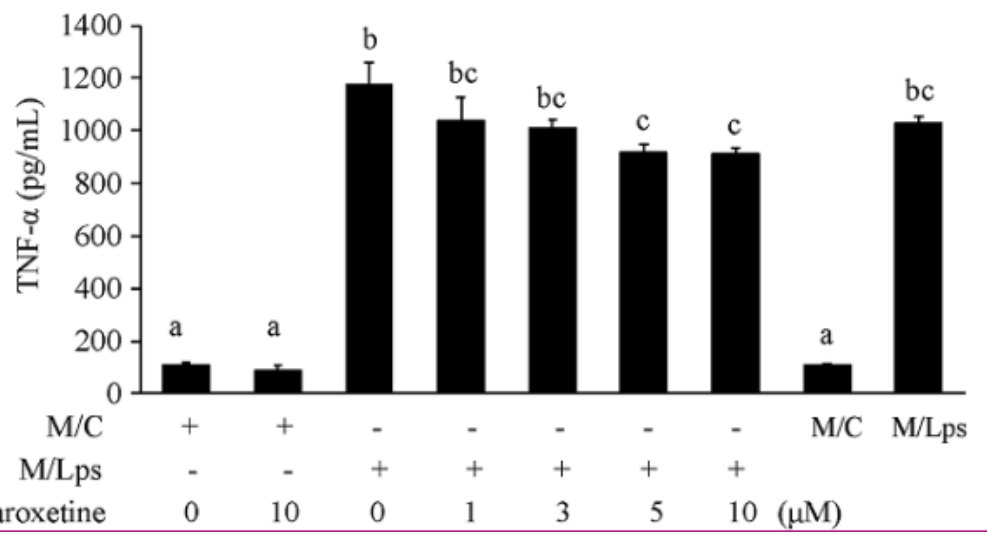

B
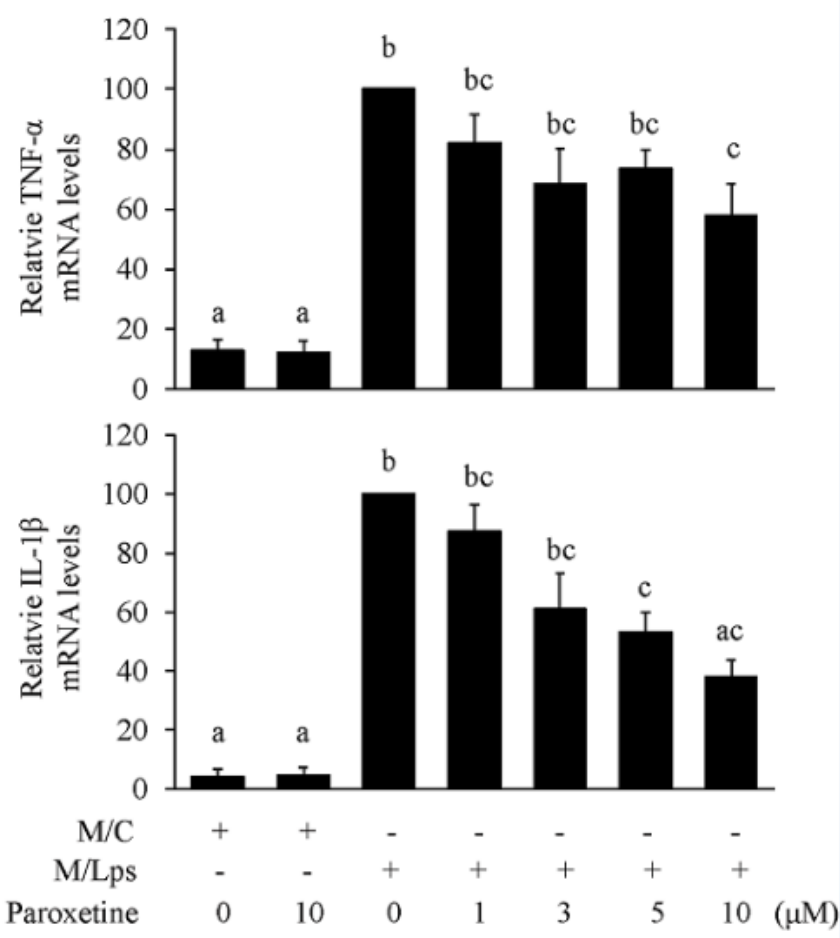

Figure 4

Paroxetine suppresses M/Lps-induced inflammatory responses in primary astrocytes. Cells were pretreated with paroxetine at different concentrations for 30 min followed by incubation with microglia conditioned media for $24 \mathrm{~h}$. (A) NO production indicated by nitrite levels in culture media. (B) Quantitative PCR analyses of TNF- $a$ and IL-1 $\beta$ expression. The mRNA levels were expressed relative to the one incubated with M/Lps alone, which was set as 100. (C) Concentrations of TNF-a in culture media. Values are means $\pm S E, n=3$ for $(A), n=4$ for $(B, C)$. Statistical comparisons were performed using one-way ANOVA, followed by the Tukey's post hoc test. Different letters indicate $p<0.05$. IL-1 $\beta$, interleukin $1 \beta ; M / C$, microglia conditioned medium without LPS stimulation; M/Lps, microglia conditioned medium preactivated with LPS; NO, nitric oxide; TNF-a, tumor necrosis factor $a$. 
A
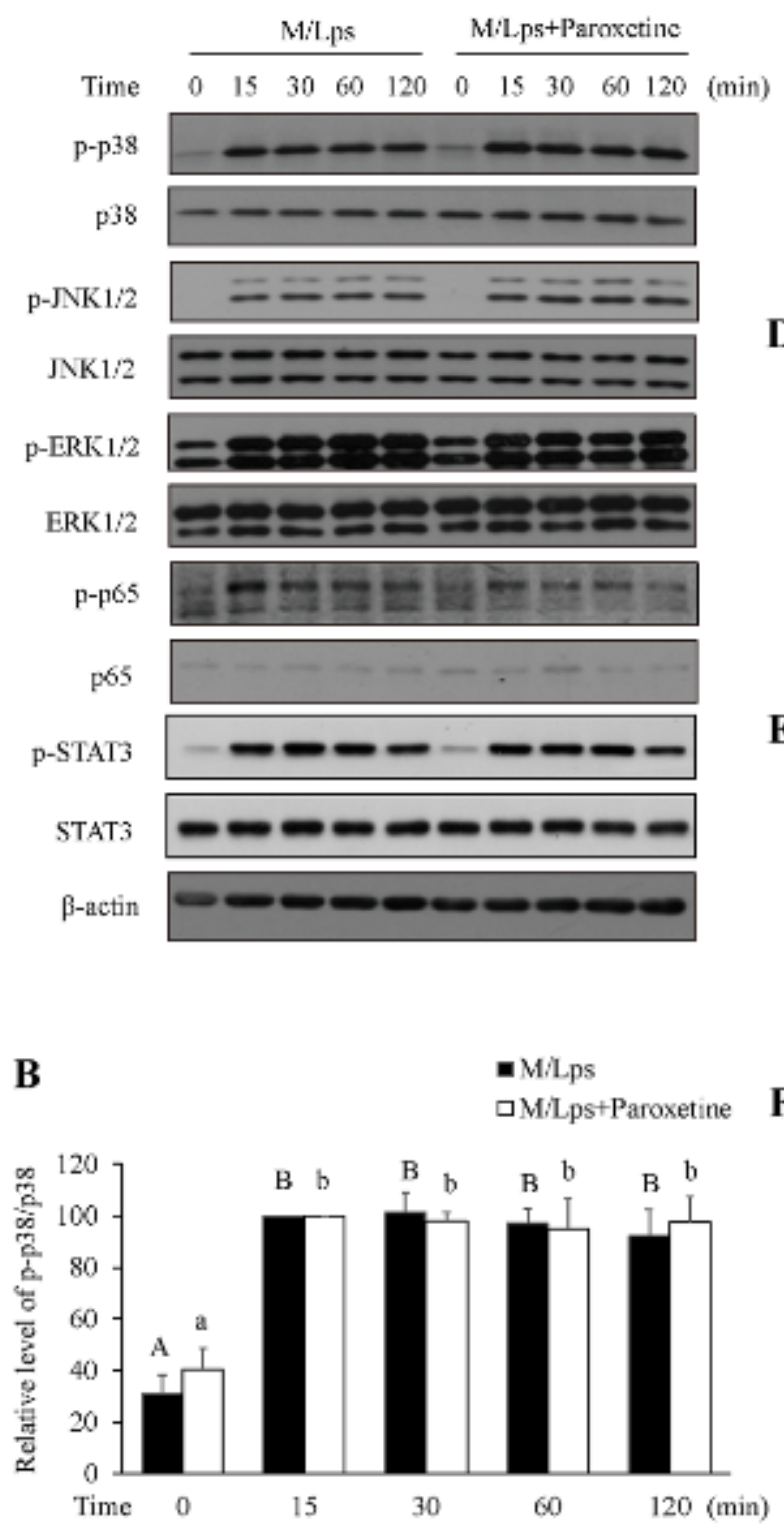

C

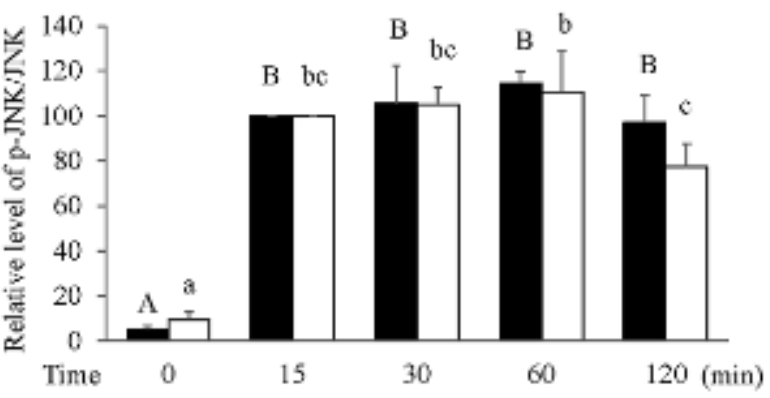

D

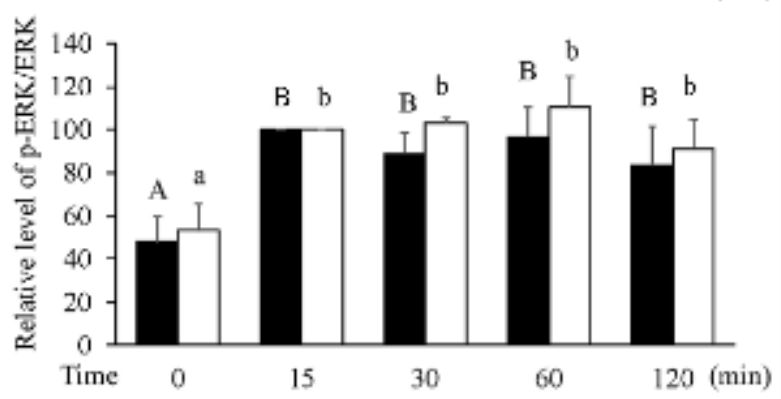

E
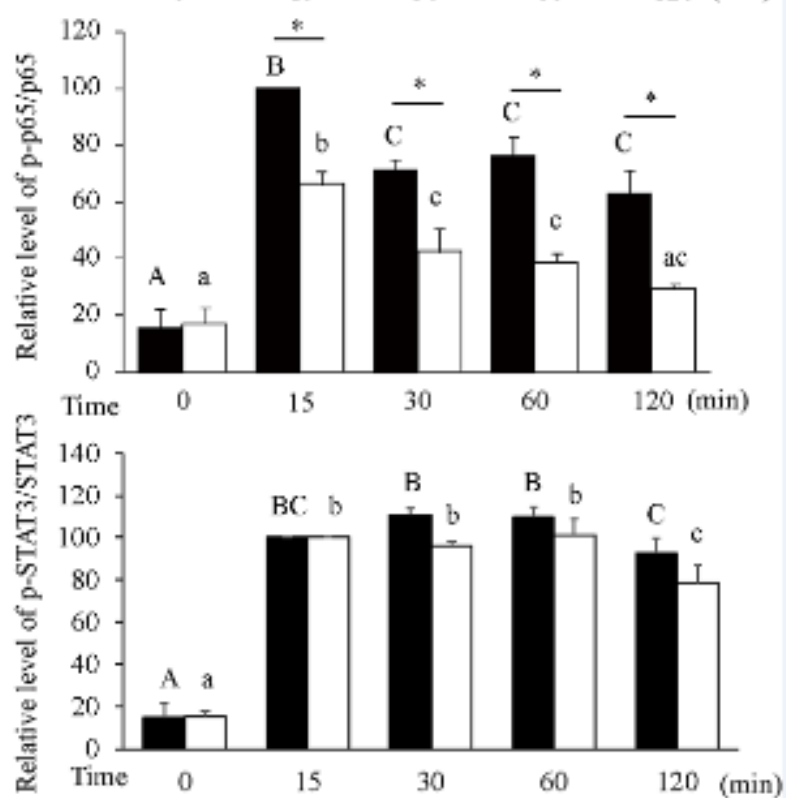

Figure 5

Effect of paroxetine on M/Lps-induced signaling activation in primary astrocytes. Cells were pretreated with or without $10 \mu \mathrm{M}$ of paroxetine for $30 \mathrm{~min}$ followed by stimulation with $\mathrm{M} / \mathrm{Lps}$ for 0-120 min. (A) Western blot analyses of p38, JNK1/2, ERK1/2, p65/NF-KB and STAT3 activation. (B-F) Levels of phosphorylation forms were quantified and normalized to their respective total levels. Values were expressed relative to the one stimulated with $\mathrm{M} / \mathrm{Lps}$ alone for $15 \mathrm{~min}$, which was set as 100 . Data are means $\pm S E, n=4$. Statistical comparisons were performed using two-way ANOVA. Different letters and * indicate $p<0.05$. M/Lps, microglia conditioned medium pre-activated with LPS. 


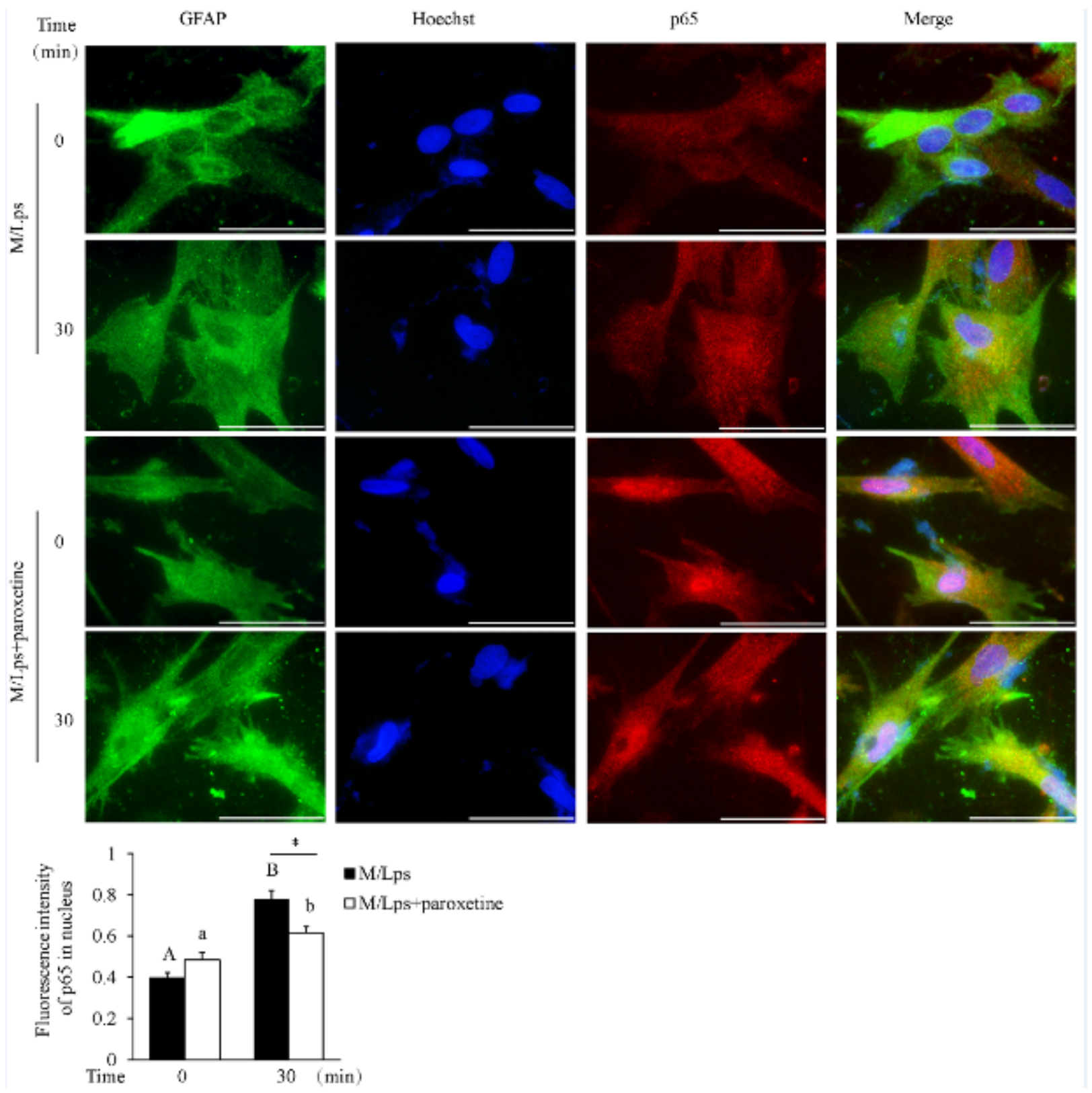

Figure 6

Effect of paroxetine on M/Lps-induced p65/NF-KB nuclear translocation in astrocytes. Cells were pretreated with or without $10 \mu \mathrm{M}$ of paroxetine for 30 min followed by stimulation with M/Lps for 30 min. (A) Representative images of p65 immunostaining. Green, GFAP; red, p65; blue, nuclei; bar size, $200 \mu \mathrm{m}$. (B) Fluorescence intensity of nuclear p65 was determined by quantifying 15 cells in multiple random fields, followed by normalization to their respective nuclei staining. Data are expressed as means $\pm S E$ from three independent experiments. Statistical comparisons were performed using two-way ANOVA. Different letters and * indicate $p<0.05$. GFAP, glial fibrillary acidic protein; $M / L p s$, microglia conditioned medium pre-activated with LPS. 

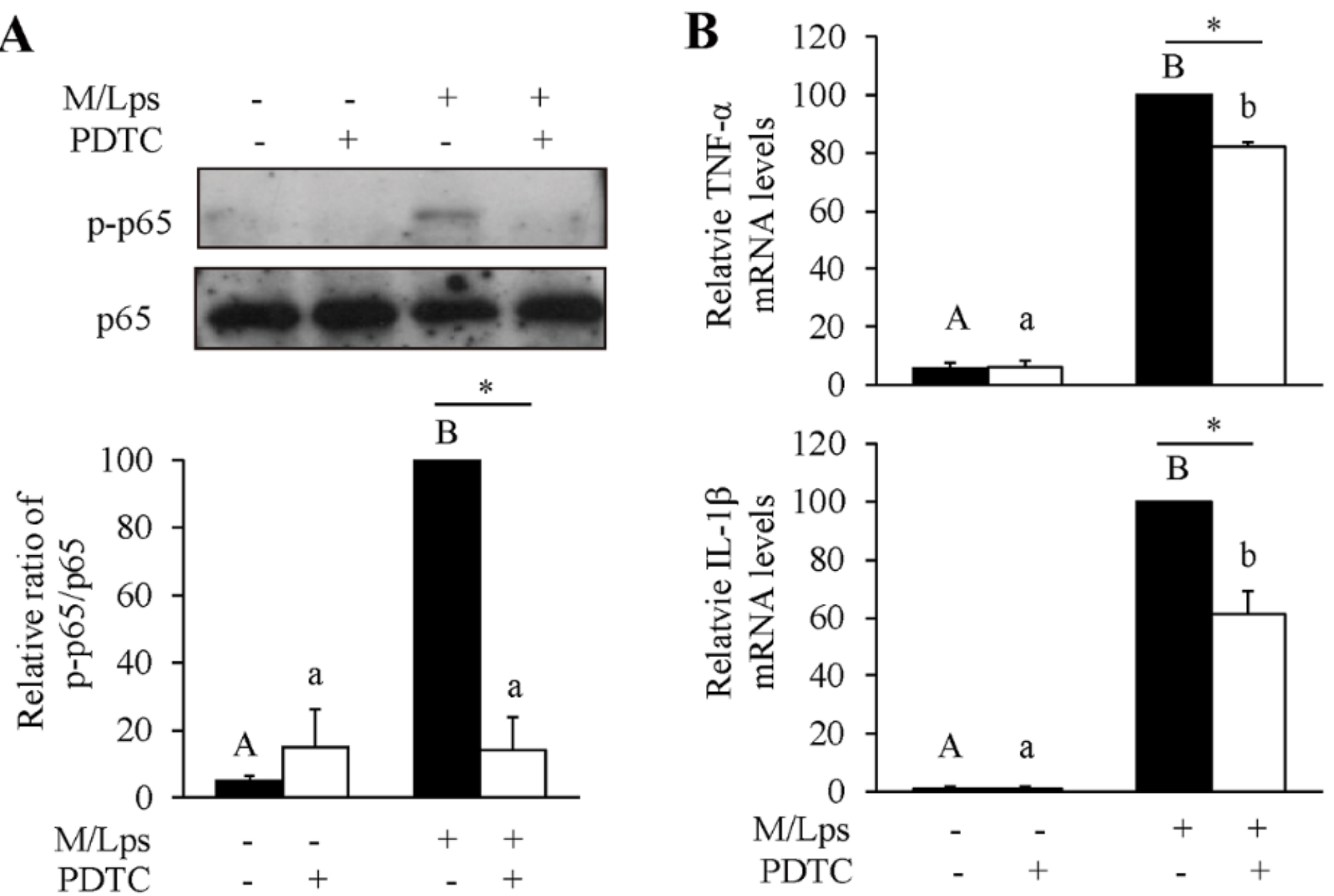

Figure 7

Inhibition of p65/NF-kB signaling on M/Lps-stimulated inflammatory responses in astrocytes. (A) Effect of PDTC on p65 activation. Cells were pretreated with $20 \mu \mathrm{M}$ of PDTC for $2 \mathrm{~h}$ prior to stimulation with M/Lps for 30 min. Levels of p-p65 were quantified and normalized to total p65 levels. (B) Effect of PDTC on M/Lps-induced mRNA expression of TNF-a and IL-1 $\beta$. Cells were pretreated with $20 \mu \mathrm{M}$ of PDTC for 2 $\mathrm{h}$ prior to stimulation with M/Lps for $24 \mathrm{~h}$. Values were expressed relative to the one stimulated with M/Lps alone, which was set as 100 . Data are means $\pm S E, n=4$. Statistical comparisons were performed using two-way ANOVA. Different letters and * indicate $p<0.05$. IL-1 $\beta$, interleukin $1 \beta ; M / C$, microglia conditioned medium without LPS stimulation; M/Lps, microglia conditioned medium pre-activated with LPS; PDTC, pyrrolidine dithiocarbamic acid; TNF-a, tumor necrosis factor a. 


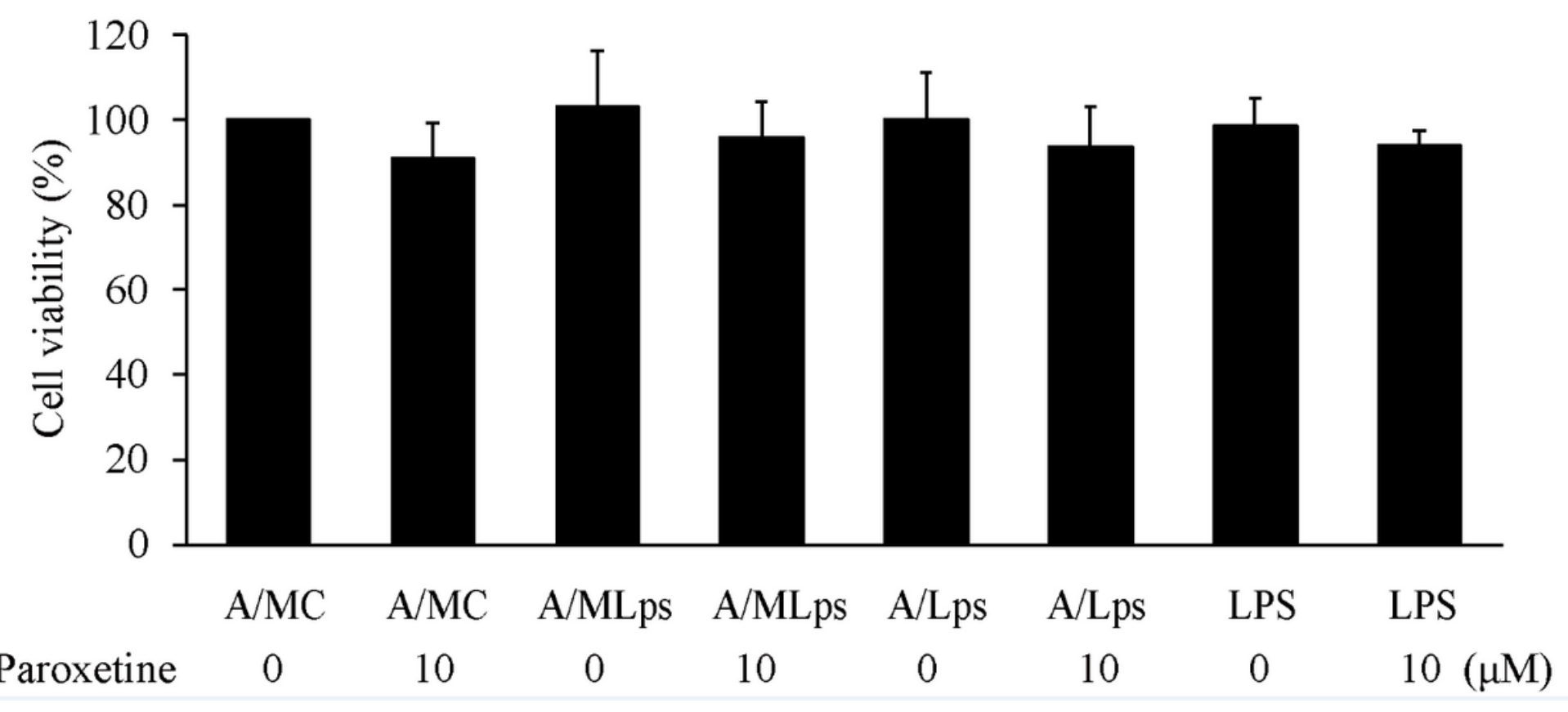

Figure 8

Effect of astrocyte conditioned media on SH-SY5Y cell survival. SH-SY5Y cells were incubated with $\mathrm{A} / \mathrm{MC}, \mathrm{A} / \mathrm{MLps}, \mathrm{A} / \mathrm{Lps}$ or LPS for $24 \mathrm{~h}$. Cell viability was expressed as percentage of the A/MC alonetreated group, which was set as $100 \%$. Values are means $\pm S E, n=3$. Statistical comparisons were performed using two-way ANOVA. A/Lps, conditioned medium of astrocytes cultured with LPS; A/MC, conditioned medium of astrocytes cultured with M/C; $A / M L p s$, conditioned medium of astrocytes cultured with M/Lps. 
$\mathbf{A}$
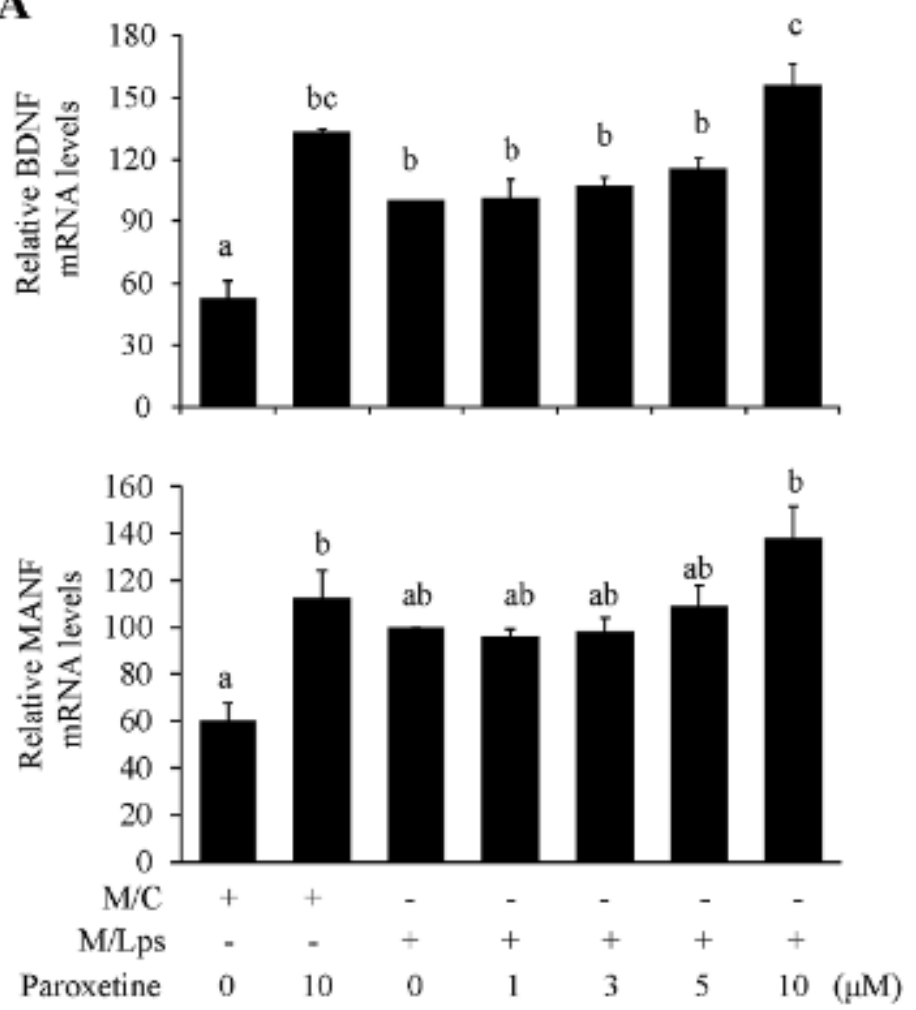

B

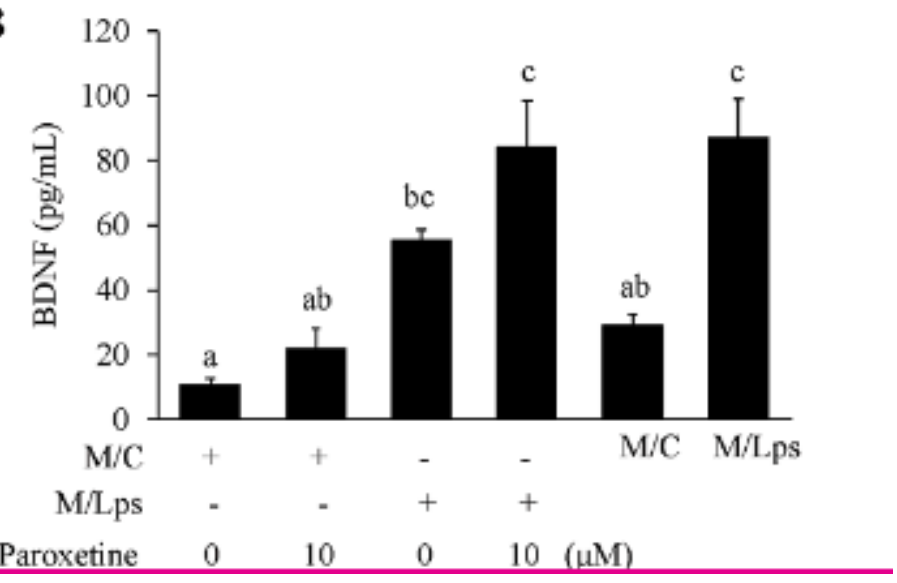

Figure 9

Effect of paroxetine on neurotrophic responses of astrocytes. Cells were pretreated with paroxetine at different concentrations for $30 \mathrm{~min}$ followed by incubation with microglia conditioned media for $24 \mathrm{~h}$. (A) Quantitative PCR analyses of BDNF and MANF expression. The mRNA levels were expressed relative to the one incubated with M/Lps alone, which was set as 100. (B) Concentrations of BDNF in culture media. Values are means $\pm S E, n=3$ for (A), $n=4$ for (B). Statistical comparisons were performed using one-way ANOVA, followed by the Tukey's post hoc test. Different letters indicate $p<0.05$. BDNF, brain derived neurotrophic factor; MANF, mesencephalic astrocyte-derived neurotrophic factor; $M / C$, microglia conditioned medium without LPS stimulation; M/Lps, microglia conditioned medium pre-activated with LPS.

\section{Supplementary Files}


This is a list of supplementary files associated with this preprint. Click to download.

- Supplementalfigures.pdf

- Highlights.docx 